\title{
Backprojection-based reconstruction and correction for distance-dependent defocus in cryoelectron microscopy
}

Kazantsev, Ivan G; Herman, Gabor T.; Cernetic, Laslo

Published in:

Biomedical Imaging: From Nano to Macro

Link to article, DOI:

10.1109/ISBI.2008.4540950

Publication date:

2008

Document Version

Publisher's PDF, also known as Version of record

Link back to DTU Orbit

Citation (APA):

Kazantsev, I. G., Herman, G. T., \& Cernetic, L. (2008). Backprojection-based reconstruction and correction for distance-dependent defocus in cryoelectron microscopy. In Biomedical Imaging: From Nano to Macro (Vol. 1-4, pp. 133-136). IEEE. https://doi.org/10.1109/ISBI.2008.4540950

\section{General rights}

Copyright and moral rights for the publications made accessible in the public portal are retained by the authors and/or other copyright owners and it is a condition of accessing publications that users recognise and abide by the legal requirements associated with these rights.

- Users may download and print one copy of any publication from the public portal for the purpose of private study or research.

- You may not further distribute the material or use it for any profit-making activity or commercial gain

- You may freely distribute the URL identifying the publication in the public portal 


\title{
BACKPROJECTION-BASED RECONSTRUCTION AND CORRECTION FOR DISTANCE-DEPENDENT DEFOCUS IN CRYOELECTRON MICROSCOPY
}

\author{
Ivan G. Kazantsev ${ }^{(1)}$, Gabor T. Herman ${ }^{(2)}$ Laslo Cernetic $^{(3)}$ \\ ${ }^{(1)}$ Materials Science Department, RIS $\varnothing$, DTU, Roskilde, Denmark \\ ${ }^{(2)}$ Dept. of Computer Science, The Graduate Center, CUNY, New York, USA \\ ${ }^{(3)}$ Dept. of Image Proc. and Comp. Graphics, University of Szeged, Szeged, Hungary \\ ${ }^{(1)}$ kazantsev1@netzero.com, ${ }^{(2)}$ gabortherman@yahoo.com, ${ }^{(3)}$ cslaszlo@inf.u-szeged.hu
}

\begin{abstract}
We study two methods of micrograph processing in cryoelectron microscopy: the defocus-gradient corrected backprojection algorithm and the correction of micrographs for the contrast transfer function based on the frequency-distance relation. Analyzing integral images produced by the methods we conclude that they are asymptotically equivalent within the framework of stationary phase approximation.
\end{abstract}

Index Terms - Electron microscopy, CTF correction

\section{INTRODUCTION}

In transmission electron microscopy (TEM), 2D projection images - called micrographs - of a 3D mass distribution (e.g., of a macromolecule) are generated in accordance with the contrast transfer function (CTF) of the electron microscope [1]. Correction for the $\mathrm{CTF}$ in the micrographs aims at estimating enhanced data that can be treated as if they were line integrals, so that algorithms from computerized tomography (CT) become applicable. Specialized filters that correct for distance-dependent distortions in 1D projections of 2D objects via the frequency-distance relation (FDR) and using a stationary phase approximation have been known for years [2]. This approach was adapted by Dubowy and Herman [3] to the $3 \mathrm{D}$ case in which the $2 \mathrm{D}$ micrographs are gathered from directions lying on a big circle around the object. In contrast to the traditional filtered backprojection technique, in which each projection is independently filtered and then backprojected, this distance-dependent CTF correction works simultaneously on the set of all projections. The extension of the $2 \mathrm{D}$ case to the special $3 \mathrm{D}$ acquisition geometry in which projection directions constitute an equatorial circle was done by using a natural $1 \mathrm{D}$ parameterization of the directions on that big circle. Further generalization to arbitrarily-oriented 2D micrographs encounters difficulties due to the complex nature of image formation in TEM and the current lack of knowledge as to how best organize the directions on the $3 \mathrm{D}$ unit sphere

\footnotetext{
This research was supported by NIH grant HL70472.
}

$S^{2}$ in a way that is appropriate for distance-dependent CTF correction.

The method DGCBP (defocus-gradient corrected backprojection) proposed by Jensen and Kornberg [4] is an alternative approach that operates on micrographs taken from arbitrary directions. The method exploits features of the forward model for TEM together with the general structure of the well-known weighted backprojection technique. However, the authors of [4] did not elaborate quantitatively how their method relates to other reconstruction/correction approaches, and they provided only a heuristic (rather than mathematical) justification as to why the method should work.

In this work we demonstrate that, for those cases for which the FDR-based CTF correction method has been mathematically derived, the heuristic DGCBP method is in some sense equivalent to it. More precisely, we show that the mathematical formulas that describe a 2D object reconstructed by the two methods from its distance-dependently distorted 1D projections in ALL directions are in fact the same. (The proof is easily generalizable to $3 \mathrm{D}$ objects to be reconstructed from 2D projections taken in ALL directions on a big circle.) This gives a further validation of the DGCBP approach for such special cases and raises the expectations of its validity in the general case. Numerical test results are presented.

\section{FORWARD MODEL OF TEM}

Let us assume that $v\left(x_{1}, x_{2}, x_{3}\right)$ is a square integrable function with an origin-centered ball as its finite support. For the projection direction vector defined by spherical coordinates $\left(\varphi_{1}, \varphi_{2}\right)$, we can calculate $v^{\varphi_{1}, \varphi_{2}}$, a rotational version of the $v$. Then a single distorted 2D projection (micrograph) $g_{\varphi_{1}, \varphi_{2}}\left(x_{1}, x_{2}\right) \equiv g\left(\varphi_{1}, \varphi_{2}, x_{1}, x_{2}\right)$ is defined as

$$
\begin{array}{r}
g_{\varphi_{1}, \varphi_{2}}\left(x_{1}, x_{2}\right)=\int_{-\infty}^{\infty} \int_{-\infty}^{\infty} \int_{-\infty}^{\infty} v^{\varphi_{1}, \varphi_{2}}\left(x_{1}^{\prime}, x_{2}^{\prime}, y\right) \times \\
h\left(x_{1}-x_{1}^{\prime}, x_{2}-x_{2}^{\prime}, y\right) d x_{1}^{\prime} d x_{2}^{\prime} d y
\end{array}
$$


where $y \equiv x_{3}$. Here, instead of rotating the acquisition system acquiring projections from different directions, we have chosen to rotate the function $v$ itself. The image formation model in TEM can be represented using the 2D Fourier transforms of the micrograph $g_{\varphi_{1}, \varphi_{2}}$, of the rotated object $v^{\varphi_{1}, \varphi_{2}}$ and of the kernel $h$ as

$$
\hat{\hat{G}}_{\varphi_{1}, \varphi_{2}}\left(k_{1}, k_{2}\right)=\int_{-\infty}^{\infty} V^{\varphi_{1}, \varphi_{2}}\left(k_{1}, k_{2}, y\right) H\left(k_{1}, k_{2}, y\right) d y
$$

where the number of hats above the $G$ aims at distinguishing between the 1-, 2-, and 3D Fourier transforms. The Fourier transform $H$ of the convolving kernel $h$ is called in TEM the Contrast Transfer Function (CTF). It can be assumed to be radially symmetric in the $\left(k_{1}, k_{2}\right)$ plane and has the form

$$
\begin{gathered}
H(k, y)=H_{C T F}(k, y) E_{\text {spat }}(k, y) E_{\text {temp }}(k), \\
H_{C T F}(k, y)=(1-a) \sin (D(k, y))-a \cos (D(k, y)), \\
D(k, y)=2 \pi \lambda k^{2}\left(-\frac{\Delta f}{2}+\frac{\lambda^{2} k^{2} C_{s}}{4}\right), \\
E_{\text {spat }}(k, y)=e^{-\pi^{2} q_{0}^{2}\left(C_{s} \lambda^{3} k^{3}-\Delta f \lambda k\right)^{2}} \\
E_{\text {temp }}(k)=e^{-\left(\frac{1}{2} \pi F_{s} \lambda k^{2}\right)^{2}}
\end{gathered}
$$

where the parameters involved are:

- $a$ is a fraction of the amplitude contrast,

$-k \equiv \sqrt{k_{1}^{2}+k_{2}^{2}}$ is a spatial frequency,

- $\lambda$ is the electron wavelength,

- $C_{s}$ is the lense spherical aberration coefficient,

- $\Delta f$ is the value of the defocus,

- $q_{0}$ is the size of the electron source,

- $F_{s}$ is the lens focal spread coefficient.

Note: the defocus $\Delta f=\Delta f(y)$ depends on the distance $y$.

A method is proposed in [3] with the following property: given distant-dependently distorted micrographs taken from directions occupying a big circle (this corresponds to the case of a constant $\varphi_{2}$ and varying $\varphi_{1}$ ), the output of the method will be corrected micrographs that are approximately $2 \mathrm{D} X$ ray transforms of the object. Denoting by $\hat{\hat{G}}\left(n, \varphi_{2}, k_{1}, k_{2}\right)$ and by $\hat{\hat{P}}\left(n, \varphi_{2}, k_{1}, k_{2}\right)$ the $3 \mathrm{D}$ Fourier transforms of the combination of all such micrographs $g\left(\varphi_{1}, \varphi_{2}, x_{1}, x_{2}\right)$ and of the corresponding true $\mathrm{X}$-ray transform $p\left(\varphi_{1}, \varphi_{2}, x_{1}, x_{2}\right)$, respectively, the stationary phase approximation implies [3] that

$$
\hat{\hat{\hat{G}}}\left(n, \varphi_{2}, k_{1}, k_{2}\right) \approx \hat{\hat{\hat{P}}}\left(n, \varphi_{2}, k_{1}, k_{2}\right) H\left(k_{1}, k_{2},-n / k_{1}\right) .
$$

Generalizations of (8) to different types of collections of projection directions (containing points on the whole of the $3 \mathrm{D}$ sphere) are not known. In many practical situations, the projection directions are spread over the sphere arbitrarily. The heuristic method of [4] is applicable even in such cases. What is more, as we now proceed to demonstrate, in the special instances when both approaches are applicable, the reconstructions produced by that method will be the same as those produced by the above-discussed CTF correction technique, at least in the limiting case of noiseless micrographs being available in all the directions on a big circle.

Let us restate this a bit more mathematically. In ordinary $\mathrm{CT}$, there are the operators $\mathcal{P}$ (projection), $\mathcal{B}$ (backprojection), and $\mathcal{D}$ (deblurring). In the mathematical limit (everything is known without noise), we know that, for any volume $v$, $v=\mathcal{D B P} v$. Let $\mathcal{C} v$ denote the distance-dependent CTF corrupted projection data of $v$. The CTF correction method estimates from such $\mathcal{C} v$ projection data $\mathcal{U C} v$ such that (hopefully) $\mathcal{U C} v=\mathcal{P} v$. If so, then $v$ can be recovered by $v=\mathcal{D} B \mathcal{U C} v$. On the other hand, DGCBP estimates from the $\mathcal{C} v$ projection data $\mathcal{V} \mathcal{C} v$ such that (hopefully) $\mathcal{V} \mathcal{C} v=\mathcal{B P} v$. If so, then $v$ can be recovered by $v=\mathcal{D} \mathcal{V C} v$. What we show in our current paper is that (again in the mathematical limit), for any $v, \mathcal{B U C} v=\mathcal{V C} v$, in those cases where both methods are applicable. (From this it follows that the two reconstructions, obtained by applying $\mathcal{D}$ to the two sides of the last equation, will also be identical.)

In case of undistorted projection data in 2D CT, the traditional notion of an integral image [5] is an integral of ridge functions

$$
c(x, y)=\int_{0}^{2 \pi} p_{\varphi}(x \cos \varphi+y \sin \varphi) d \varphi,
$$

where

$$
p_{\varphi}(x) \equiv p(\varphi, x)=\int_{-\infty}^{\infty} v^{\varphi}(x, y) d y
$$

is a true X-ray projection, and rotational version $v^{\varphi}$ of $v$ is

$$
v^{\varphi}(x, y)=v(x \cos \varphi-y \sin \varphi, x \sin \varphi+y \cos \varphi) .
$$

Then, in our symbolic notation, the set of all $p_{\varphi}$ is what is referred to above as $\mathcal{P} v$, and the $c$ in (9) is in fact $\mathcal{B P} v$.

\section{ANALYSIS OF INTEGRAL IMAGES}

In what follows we use the simplified 2D formulation of the TEM model. Generalization to the $3 \mathrm{D}$ case with equatorial orbits is straightforward. The 2D analog of the forward model (1) is

$$
g(\varphi, x) \equiv g_{\varphi}(x)=\int_{-\infty}^{\infty} \int_{-\infty}^{\infty} v^{\varphi}\left(x^{\prime}, y\right) h\left(x-x^{\prime}, y\right) d x^{\prime} d y
$$

The set of all $g_{\varphi}$ is what is referred to above as $\mathcal{C} v$. The 2D version of model (2) is then

$$
\hat{G}_{\varphi}(k)=\int_{-\infty}^{\infty} V^{\varphi}(k, y) H(k, y) d y .
$$




\subsection{Defocus-gradient corrected backprojection}

Aiming at defocus elimination [4], defocus-gradient corrected backprojection (DGCBP) uses a generalized version $b_{\varphi}$ of ridge functions, defined by

$$
b_{\varphi}(x, y)=\mathcal{F}^{-1}\left[\hat{B}_{\varphi}(k, y)\right]=\int_{-\infty}^{\infty} \hat{B}_{\varphi}(k, y) e^{2 \pi i k x} d k,
$$

where explicit dependence upon the variable $y$ arises due to compensative filtering that depends on $y$ and is given by

$$
\hat{B}_{\varphi}(k, y)=\hat{G}_{\varphi}(k) / H(k, y)=\hat{G}_{\varphi}(k) H^{-1}(k, y),
$$

assuming that the inverse $H^{-1}$ exists and is well-defined. Integration of the generalized ridge functions $b_{\varphi}(x, y)$ provides us with the generalized integral image

$$
w(x, y)=\int_{0}^{2 \pi} b_{\varphi}(x \cos \varphi+y \sin \varphi,-x \sin \varphi+y \cos \varphi) d \varphi .
$$

This $w$ is the $\mathcal{V C} v$ of Section 2. The expectation that $w=$ $\mathcal{V C} v$ is in fact $\mathcal{B P} v$ is based on the hope that distortions in the slices above and below any particular slice would cancel out due to the rotation of the projection direction.

We are going to explore how the generalized integral image $w(x, y)$ of the DGCBP method is related to the approximation $c^{*}(x, y)$ that is provided by CTF correction for the integral image $c(x, y)$. For that purpose, let the original image be the delta function

$$
v(x, y)=\delta\left(x_{0}-x\right) \delta\left(y_{0}-y\right),
$$

centered at a point $\left(x_{0}, y_{0}\right) \in R^{2}$. We wish to compute its generalized integral image. It can be shown that the micrograph of $v$ is

$g_{\varphi}(x)=h\left(x-x_{0} \cos \varphi-y_{0} \sin \varphi,-x_{0} \sin \varphi+y_{0} \cos \varphi\right)$,

and so the forward Fourier transform of $g_{\varphi}(x)$ is

$\hat{G}_{\varphi}(k)=H\left(k,-x_{0} \sin \varphi+y_{0} \cos \varphi\right) e^{-2 \pi i k\left(x_{0} \cos \varphi+y_{0} \sin \varphi\right)}$.

Recalling that a generalized ridge function (14) is

$$
b_{\varphi}(x, y)=\int_{-\infty}^{\infty} \hat{G}_{\varphi}(k) H^{-1}(k, y) e^{2 \pi i k x} d k,
$$

and substituting (19) and (20) into (16) we obtain that the generalized integral image for the delta function is

$$
\begin{array}{r}
w(x, y)=\int_{0}^{2 \pi} \int_{-\infty}^{\infty} e^{2 \pi i k\left[\left(x-x_{0}\right) \cos \varphi+\left(y-y_{0}\right) \sin \varphi\right]} \times \\
\frac{H\left(k,-x_{0} \sin \varphi+y_{0} \cos \varphi\right)}{H(k,-x \sin \varphi+y \cos \varphi)} d k d \varphi .
\end{array}
$$

\subsection{CTF correction of micrographs}

We can represent the true X-ray projection $p_{\varphi}$ in the form:

$$
p(\varphi, x)=\sum_{n=-\infty}^{\infty} \int_{-\infty}^{\infty} \hat{\hat{P}}(n, k) e^{2 \pi i(k x+n \varphi)} d k
$$

where the term $\hat{\hat{P}}(n, k)$ is not available in practice. It is estimated by a correction of $\hat{\hat{G}}(n, k)$, the 2D Fourier transform of the corrupted projection data $g(\varphi, x)$. The correction is based on the FDR principle [2] (see also (26)), which implies

$$
\hat{\hat{G}}(n, k) \approx \hat{\hat{P}}(n, k) H(k,-n / k),
$$

which is known as the CTF correction equation. Then substituting (22) and (23) into (9) we obtain

$$
\begin{aligned}
c(x, y) \approx c^{*}(x, y) \equiv \int_{0}^{2 \pi} \sum_{n=-\infty}^{\infty} \int_{-\infty}^{\infty} \frac{\hat{\hat{G}}(n, k)}{H(k,-n / k)} \times \\
e^{2 \pi i[k(x \cos \varphi+y \sin \varphi)+n \varphi]} d k d \varphi .
\end{aligned}
$$

This $c^{*}$ is the $\mathcal{B U C} v$ in Section 2.

The last integral in (24) can be modified using the stationary phase approximation: the largest contribution from the highly oscillating integrand originates from those parts of its domain for which the phase $k(x \cos \varphi+y \sin \varphi)+n \varphi$ changes most slowly. These parts are segments within which a stationary phase occurs, i.e., which contain values of $\varphi$ that satisfy

$$
\frac{\partial}{\partial \varphi}[k(x \cos \varphi+y \sin \varphi)+n \varphi]=0,
$$

or, in the form of the frequency-distance relation,

$$
-x \sin \varphi+y \cos \varphi=-n / k .
$$

Assuming that $1 / H(k,-n / k)$ is smooth enough, we can replace $H(k,-n / k)$ in (24) by $H(k,-x \sin \varphi+y \cos \varphi)$.

Now we are going to prove that if $c^{*}(x, y)$ is defined by (24) for the delta function $v$ defined by (17), then $c^{*}$ equals the associated $w$ as defined by (21). For this $v$, we calculate $\hat{\hat{G}}(n, k)$, by taking the Fourier transform of the function defined in (19) with respect to its angular variable, and get

$$
\begin{aligned}
\hat{\hat{G}}(n, k)= & \int_{0}^{2 \pi} H\left(k,-x_{0} \sin \psi+y_{0} \cos \psi\right) \times \\
& e^{-2 \pi i k\left(x_{0} \cos \psi+y_{0} \sin \psi\right)} e^{-2 \pi i n \psi} d \psi .
\end{aligned}
$$

Substitution of (27) into (24) produces after a reversal of the 
order of integrations and the sum

$$
\begin{gathered}
c^{*}(x, y)=\int_{0}^{2 \pi} \int_{-\infty}^{\infty} H\left(k,-x_{0} \sin \psi+y_{0} \cos \psi\right) \times \\
{\left[\sum_{n=-\infty}^{\infty} \int_{0}^{-2 \pi i k\left(x_{0} \cos \psi+y_{0} \sin \psi\right)} \frac{e^{2 \pi i[k(x \cos \varphi+y \sin \varphi)+n(\varphi-\psi)]}}{H(k,-x \sin \varphi+y \cos \varphi)} d \varphi\right] d k d \psi}
\end{gathered}
$$

It is not difficult to show that the expression in brackets in (28) equals $\frac{e^{2 \pi i k(x \cos \psi+y \sin \psi)}}{H(k,-x \sin \psi+y \cos \psi)}$ and (28) thus reduces to the form of (21). This proves that, for a delta function at an arbitrary location, the $c^{*}(x, y)$ obtained from a complete set of CTFcorrected noiseless but CTF-corrupted micrographs is equivalent (within the framework of stationary phase approximation) to the $w(x, y)$ obtained by the DGCBP method (21). By the validity of CTF correction, they are both equivalent to

$$
c(x, y) \approx 2 / \sqrt{\left(x-x_{0}\right)^{2}+\left(y-y_{0}\right)^{2}} \equiv 2 / r
$$

which is the ideal (in case of noiseless 2D CT projection data) radially symmetric integral image [5] with profile $2 / r$.

\section{NUMERICAL EXPERIMENTS}

We compare the integral image $w(x, y)$ (Fig. 1(a)), produced numerically by the DGCBP technique for $\left(x_{0}, y_{0}\right)=(0,0)$ using formula (21), and the ideal integral image (29) with profile $2 / r$ (Fig. 1(b)). For the inverse of $H$ we used

$$
H^{+}(k, y)=H(k, y) /\left[H(k, y) H(k, y)+\alpha k^{m}\right]
$$

where $\alpha=0.000001$ and $m=2$. The parameters are:

$$
\begin{aligned}
& -a=0 ; \\
& -\lambda=0.033487 \AA ; \\
& \text { - } C_{s}=22,000,000 \AA ; \\
& \text { - } \Delta f \in[1500,2500](\text { in } \AA \text { ) is linear defocus; } \\
& \text { - } q_{0}=0.25 \cdot 10^{-3} / \lambda ; \\
& \text { - } F_{s}=141.35 \AA .
\end{aligned}
$$

We show also a 3D DGCBP reconstruction from 5, $000 \mathrm{mi}-$ crographs taken from random directions (Fig. 1(d)). These were numerically generated for a test object of seven spheres (Fig. 1(c)).

\section{DISCUSSION}

In this work we have shown that DGCBP and CTF correction, when both applicable, are likely to produce similar integral images. The behavior of the two methods was illustrated on two simple examples. It appears to be close to that of CT.

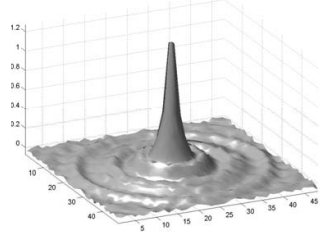

(a) Plot of $w(x, y)$

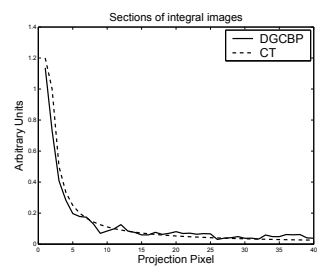

(b) Sections of $w(x, y)$ and $2 / r$

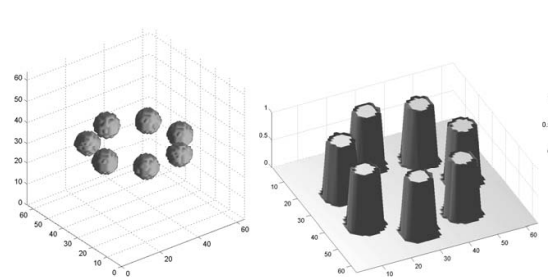

(c) Test object

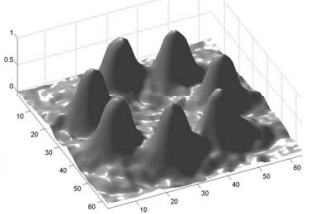

(d) 3D DGCBP

Fig. 1. (a) Plot of integral image of the delta function by the 2D DGCBP algorithm; (b) two profiles (DGCBP and CT) of integral images of the delta function; (c) 3D view (left) and plot of test object's central slice (right); (d) Plot of central slice of the 3D DGCBP reconstruction.

\section{ACKNOWLEDGEMENT}

This work was done during the stay of the first and the third authors with the Digital Imaging and Graphics group of the Graduate Center of the City University of New York. The first author gratefully acknowledges the Danish National Research Foundation for supporting the Center for Fundamental Research: Metal Structures in Four Dimensions, within which this work was finalized.

\section{REFERENCES}

[1] J. Frank, Three-Dimensional Electron Microscopy of Macromolecular Assemblies, Oxford University Press, 2006.

[2] P.R. Edholm, R.M. Lewitt, and B. Lindholm, "Novel properties of the Fourier decomposition of the sinogram," in SPIE Proc Physics Eng Computer Multidimensional Image Processing. SPIE, 1986, vol. 0671, pp. 8-18.

[3] J.N. Dubowy and G.T. Herman, "An approach to the correction of distance-dependent defocus in electron microscopic reconstruction," in Proceedings of the IEEE International Conference on Image Processing. IEEE, 2005, vol. 3, pp. 748-751.

[4] G.J. Jensen and R.D. Kornberg, "Defocus-gradient corrected back-projection," Ultramicroscopy, vol. 84, pp. 57-64, 2000.

[5] F. Natterer and F. Wübbeling, Mathematical Methods in Image Reconstruction, SIAM, 2001. 